\title{
Improving Interoperability Using Query Interpretation in Semantic Vector Spaces
}

\author{
Anthony Ventresque ${ }^{1}$, Sylvie Cazalens ${ }^{1}$, Philippe Lamarre ${ }^{1}$, \\ and Patrick Valduriez ${ }^{2}$ \\ LINA, University of Nantes \\ FirstName.LastNameQuniv-nantes.fr \\ INRIA and LINA, University of Nantes \\ Patrick.Valduriez@inria.fr
}

\begin{abstract}
In semantic web applications where query initiators and information providers do not necessarily share the same ontology, semantic interoperability generally relies on ontology matching or schema mappings. Information exchange is then not only enabled by the established correspondences (the "shared" parts of the ontologies) but, in some sense, limited to them. Then, how the "unshared" parts can also contribute to and improve information exchange ? In this paper, we address this question by considering a system where documents and queries are represented by semantic vectors. We propose a specific query expansion step at the query initiator's side and a query interpretation step at the document provider's. Through these steps, unshared concepts contribute to evaluate the relevance of documents wrt. a given query. Our experiments show an important improvement of retrieval relevance when concepts of documents and queries are not shared. Even if the concepts of the initial query are not shared by the document provider, our method still ensures $90 \%$ of the precision and recall obtained when the concepts are shared.
\end{abstract}

\section{Introduction}

In semantic web applications where query initiators and information providers do not necessarily share the same ontology, semantic interoperability generally relies on ontology matching or schema mappings. Several works in this domain focus on what (i.e. the concepts and relations) the peers share 918. This is quite important because, obviously if nothing is shared between the ontologies of two peers, there is a little chance for them to understand the meaning of the information exchanged. However, no matter how the shared part is obtained (through consensus or mapping), there might be concepts (and relations) that are not consensual, and thus not shared. The question is then to know whether the unshared parts can still be useful for information exchange.

In this paper, we focus on semantic interoperability and information exchange between a query initiator $p_{1}$ and a document provider $p_{2}$, which use different ontologies but share some common concepts. The problem we address is to find documents which are relevant to a given query although the documents and the

S. Bechhofer et al.(Eds.): ESWC 2008, LNCS 5021, pp. 539-553 2008.

(C) Springer-Verlag Berlin Heidelberg 2008 
query may be both represented with concepts that are not shared. This problem is very important because in semantic web applications with high numbers of participants, the ontology (or ontologies) is rarely entirely shared. Most often, participants agree on some part of a reference ontology to exchange information and internally, keep working with their own ontology [1822].

We represent documents and queries by semantic vectors [25], a model based on the vector space model [1] using concepts instead of terms. Although there exist other, richer representations (conceptual graphs for example), semantic vectors are a common way to represent unstructured documents in information retrieval. Each concept of the ontology is weighted according to its representiveness of the document. The same is done for the query. The resulting vector represents the document (respectively, the query) in the n-dimensional space formed by the $n$ concepts of the ontology. Then the relevance of a document with respect to a query corresponds to the proximity of the vectors in the space.

In order to improve information exchange beyond the "shared part" of the ontologies, we promote both query expansion (at the query initiator's side) and query interpretation (at the document provider's side). Query expansion may contribute to weight linked shared concepts, thus improving the document provider's understanding of the query. Similarly, by interpreting an expanded query with respect to its own ontology (i.e. by weighting additional concepts of its own ontology), the document provider may find additional related documents for the query initiator that would not be found by only using the matching concepts in the query and the documents. Although the basic idea of query expansion and interpretation is simple, query interpretation is very difficult because it requires to precisely weight additional concepts given some weighted shared ones, while the whole space (i.e. the ontology) and similarity measures change.

In this context, our contributions are the following. First, we propose a specific query expansion method. Its property is to keep separate the results of the propagation from each central concept of the query, thus limiting the noise due to inaccurate expansion. Second, given this expansion, we define the relevance of a document. Its main, original characteristic is to require the document vector to be requalified with respect to the expanded query, the result being called image of the document. Third, a main contribution is the definition of query interpretation which enables the expanded query to be expressed with respect to the provider's ontology. Fourth, we provide two series of experiments with still very good results although few concepts are shared.

This paper is organized as follows. Section 2 gives preliminary definitions. Section 3 presents our query expansion method and the image based relevance of a document. For simplicity, we assume a context of shared ontology. This assumption is relaxed after in Section 4, where we consider the case where the query initiator and the document provider use different ontologies and present the query interpretation. Section 5 discusses the experiments and their results. The two last sections are respectively devoted to related work and conclusion. 


\section{Preliminary Definitions}

We define an ontology as a set of concepts together with a set of relations between these concepts. In our experiments, we consider an ontology with only one relation: the is-a relation (specialization link). This does not restrict the generality of our relevance computation. Indeed, the presence of several relations only affects the definition of the similarity of a concept wrt. another. A semantic vector $\overrightarrow{v_{\Omega}}$ is an application defined on the set of concepts $\mathcal{C}_{\Omega}$ of the ontology $\Omega$ : $\forall c \in \mathcal{C}_{\Omega}, \overrightarrow{v_{\Omega}}: c \rightarrow[0 . .1]$. A popular way to compute the relevance of a document is to use the cosine-based proximity of the document and query vectors in the space [19]. The problem with cosine is the independence of dimensions : a query on concept $c_{i}$ and a document on concept $c_{j}$ very close from $c_{i}$ could not match. Query expansion is generally used to express these links between concepts, by propagating initial weights on other linked concepts. To define a query expansion, we need a similarity function [11] which expresses how much a concept is similar to another within the ontology $: \operatorname{sim}_{c}: \mathcal{C}_{\Omega} \rightarrow[0,1]$, is a similarity function iff $\operatorname{sim}_{c}(c)=1$ and $0 \leq \operatorname{sim}_{c}\left(c_{j}\right)<1$ for all $c_{j} \neq c$ in $\mathcal{C}_{\Omega}$. Then, propagation from a central concept $c$ of weight $v$ assigns a weight to every value of similarity with $c$.

Definition 1 (Propagation function). Let $c$ be a concept of $\Omega$ valued by $v$; and let sim $_{c}$ be a similarity function.

$$
\begin{aligned}
& \text { A function } \mathcal{P} f_{c}: \mid \begin{array}{l}
{[0 . .1] \quad \mapsto[0 . .1] \quad \text { is a propagation function from } c \text { iff }} \\
\operatorname{sim}_{c}\left(c^{\prime}\right) \rightarrow \mathcal{P} f_{c}\left(\operatorname{sim}_{c}\left(c^{\prime}\right)\right)
\end{array} \\
& -\mathcal{P} f_{c}\left(\operatorname{sim}_{c}(c)\right)=v, \text { and } \\
& -\forall c_{k}, c_{l} \in \mathcal{C}_{\Omega} \operatorname{sim}_{c}\left(c_{k}\right) \leq \operatorname{sim}_{c}\left(c_{l}\right) \Rightarrow \mathcal{P} f_{c}\left(\operatorname{sim}_{c}\left(c_{k}\right)\right) \leq \mathcal{P} f_{c}\left(\operatorname{sim}_{c}\left(c_{l}\right)\right)
\end{aligned}
$$

Among different types of propagation functions those inspired by the membership functions used in fuzzy logic work fine (see Figure 1) in our experiments. It is defined by three parameters $v$ (weight of the central concept), $l_{1}$ (similarity value until which concepts have the same weight $: v$ ) and $l_{2}$ (similarity value until which concepts have non zero weight) such that, $\forall x=\operatorname{sim}_{c}\left(c^{\prime}\right), c^{\prime} \in c_{\Omega}$ :

$$
\mathcal{P} f_{c}(x)=f_{v, l_{1}, l_{2}}(x)=\mid \begin{array}{ll}
v & \text { if } x \geq l_{1} \\
\frac{v}{l_{1}-l_{2}} x+\frac{l_{2} \times v}{l_{1}-l_{2}} & \text { if } l_{1}>x>l_{2} \\
0 & \text { if } l_{2} \geq x
\end{array}
$$

\section{Query Expansion and Image Based Relevance}

In this section, we present our method to compute the relevance of a document wrt a query. For the sake of simplicity, we assume that the query initiator and the document provider use the same ontology. However, they can still differ on the similarity measures and the propagation functions. First, we compute a query expansion, and then an image of a document vector to compute the relevance of the document wrt. a query in a single space. 


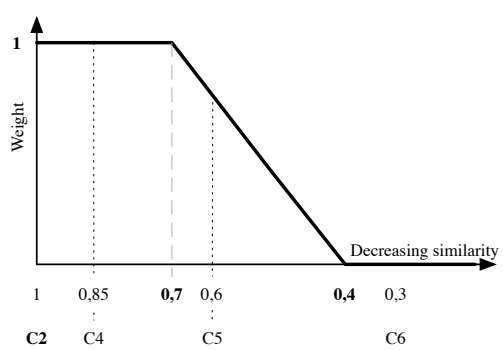

Fig. 1. Example of a propagation function $f_{1,0.7,0.4}$ with central concept $c_{2}$

To our knowledge, most query expansion methods propagate the weight of each weighted concept in the same vector, thus directly adding the expanded terms in the original vector 13 . When a concept is involved in several propagations conducted from different central concepts, an aggregation function (e.g. the maximum) is used. We call this kind of method "rough" propagation. Although its results are not bad, such a propagation has some drawbacks among which a possible unbalance of the relative importance of the initial concepts [16]. First, let us denote by $\mathcal{C}_{\vec{q}}$ the set of the central concepts of query $\vec{q}$, i.e. those weighted concepts which represent the query. To keep separate the effects of different propagations, each central concept of $\mathcal{C}_{\vec{q}}$ is semantically enriched by propagation, in a separate vector.

Definition 2 (Semantically Enriched Dimension). Let $\vec{q}$ be a query vector and let $c$ be a concept in $\mathcal{C}_{\vec{q}}$. A semantic vector $\overrightarrow{s e d}_{c}$ is a semantically enriched dimension, iff $\forall c^{\prime} \in \mathcal{C}_{\Omega}, \overrightarrow{\operatorname{sed}}_{c}\left[c^{\prime}\right] \leq \overrightarrow{\operatorname{sed}}_{c}[c]$.

Definition 3 (Expansion of a query). Let $\vec{q}$ be a query vector. An expansion of $\vec{q}$, noted $\mathcal{E}_{\vec{q}}$ is a set defined by:

$\mathcal{E}_{\vec{q}}=\left\{\overrightarrow{\operatorname{sed}}_{c}: c \in \mathcal{C}_{\vec{q}}, \forall c^{\prime} \in \mathcal{C}_{\Omega}, \overrightarrow{\operatorname{sed}}_{c}\left[c^{\prime}\right]=\mathcal{P} f_{c}\left(c^{\prime}\right)\right\}$

Figure 2 illustrates the expansion of a query $\vec{q}$ with two weighted concepts $c_{4}$ and $c_{7}$. It contains two semantically enriched dimensions. In dimension $\overrightarrow{s e d}_{c_{7}}$, concept $c_{7}$ has the same value as in the query. The weight of $c_{7}$ has been propagated on $c_{3}, c_{11}$ and $c_{6}$ according to their similarity with $c_{7}$. The other dimension is obtained from $c_{4}$ in the same way.

The expanded query is composed of several semantic vectors (the SEDs). Our aim is then to transform the semantic vector of a document, $\vec{d}$, in an image through the expanded query, i.e. to characterize the document wrt. each central concept $c$ (dimension) of the query, as far as it has concepts related to $c$, in particular even if $c$ is not initially weighted in $\vec{d}$. Given a SED $\overrightarrow{s e d}_{c}$, we aim at valuating $c$ in the image of the document $\vec{d}$ according to the relevance of $\vec{d}$ to $\overrightarrow{s e d}_{c}$. To evaluate the impact of $\overrightarrow{s e d}_{c}$ on $\vec{d}$ we consider the product of the respective values of each concept in $\overrightarrow{s e d}_{c}$ and $\vec{d}$. Intuitively, all the concepts 


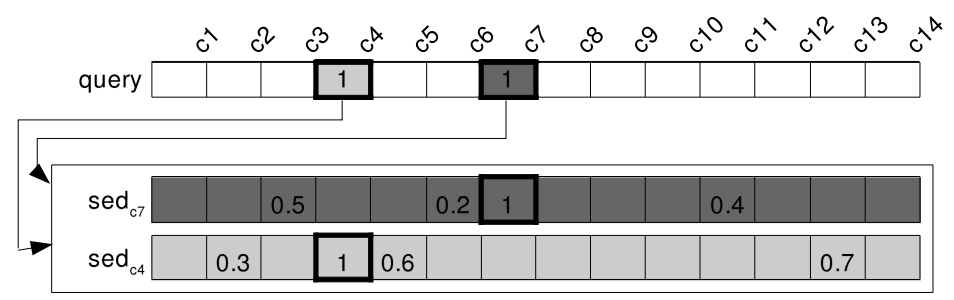

Fig. 2. A query expansion composed of 2 semantically enriched dimensions

of the document which are linked to $c$ through $\overrightarrow{s e d}_{c}$ have a nonnull value. The image of $\vec{d}$ keeps track of the best value assigned to one of the linked concepts if it is better than $\vec{d}[c]$, which is the initial value of $c$. This process is repeated for each SED of the query. Algorithm 1.1 gives the computation of the image of document $\vec{d}$, noted $\vec{i}_{d}$. This algorithm ensures that all the central concepts of the initial query vector are also weighted in the image of the document as far as the document is related to them. Wrt. the query, the image of the document is more accurate because it enforces the documents characterization over each dimension of the query. However, in the image, we keep unchanged the weights of the concepts which are not linked to any concept of the query (i.e. which are not weighted in any SED). The example of Figure 3 illustrates how the image of a document is computed.

Algorithm 1.1. Image of a document wrt a query

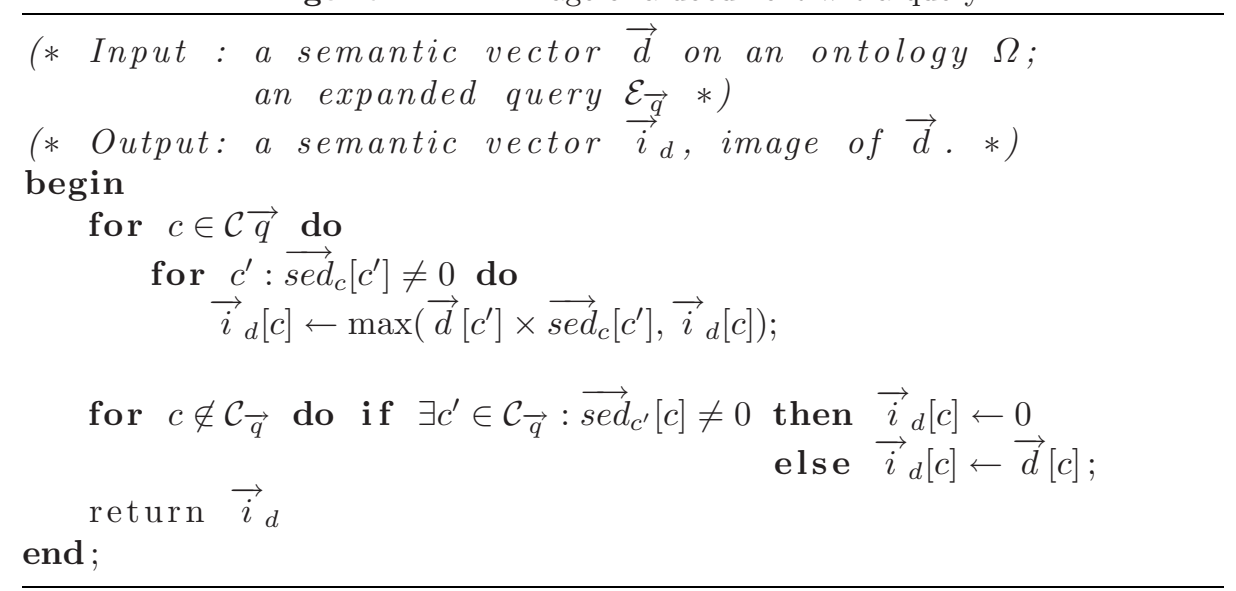

We define the relevance of $\vec{d}$ wrt. $\vec{q}$ by $\cos \left(\vec{i}_{d}, \vec{q}\right)$. Considering the image enables to take into account the documents that have concepts linked to those of the query. Using a cosine, and thus the norm of the vectors, assigns a lower importance to the documents with an important norm, which are often very general. 


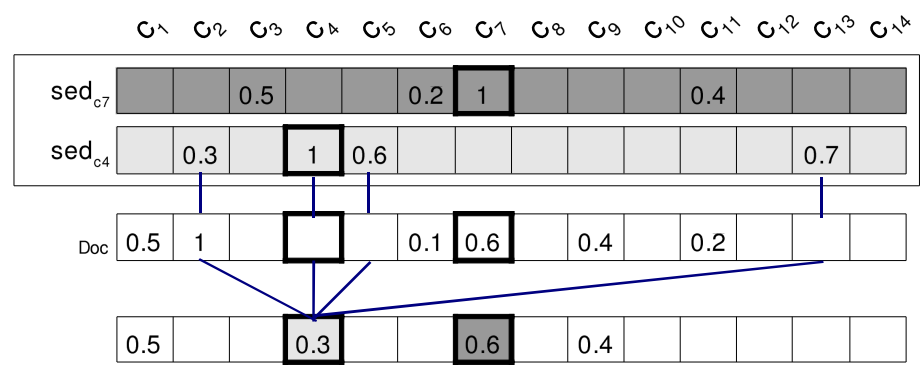

Fig. 3. Obtaining the image of a document

\section{Relevance in the Context of Unshared Concepts}

In this section, we assume that the query initiator and the document provider do not use the same ontology. We follow the approach adopted in Section 3, using a query expansion at the query initiator's side and the computation of the image of the document at the provider's side. But things get complicated by the fact that the query initiator and the document provider do not use the same vector space. An additional step is needed in order to evaluate relevance in a same and single space. Thus, we introduce a query interpretation step at the provider's side.

\subsection{Computing Relevance: Overview}

As shown in Figure 4, the query initiator, denoted by $p_{1}$, works within the context of ontology $\Omega_{1}$, while the document provider, noted $p_{2}$, works with ontology $\Omega_{2}$. Through its semantic indexing module, the query initiator (respectively the document provider) produces the query vector (respectively the document vector), which is expressed on $\Omega_{1}$ (respectively $\Omega_{2}$ ). Both $p_{1}$ and $p_{2}$ also have their own way of computing both the similarity and the propagation.

We assume that the query initiator and the document provider share some common concepts, meaning that each of them regularly, although may be not often, runs an ontology matching algorithm. Ontology matching results in an alignment between two ontologies, which is composed of a (non empty) set of correspondences with some cardinality and, possibly some meta-data [4]. A correspondence establishes a relation (equivalence, subsumption, disjointness...) between some entities (in our case, concepts), with some confidence measure. Each correspondence has an identifier. In this paper, we only consider the equivalence relation between concepts and those couples of equivalent concepts of which confidence measure is above some threshold. We call them the shared concepts. For simplicity, when there is an equivalence, we make no distinction between the name of the given concept at $p_{1}$ 's, its name at $p_{2}$ 's, and the identifier of the correspondence, which all refer to the same concept. Hence, the set of shared concepts is denoted by $\mathcal{C}_{\Omega_{1}} \cap \mathcal{C}_{\Omega_{2}}$. 


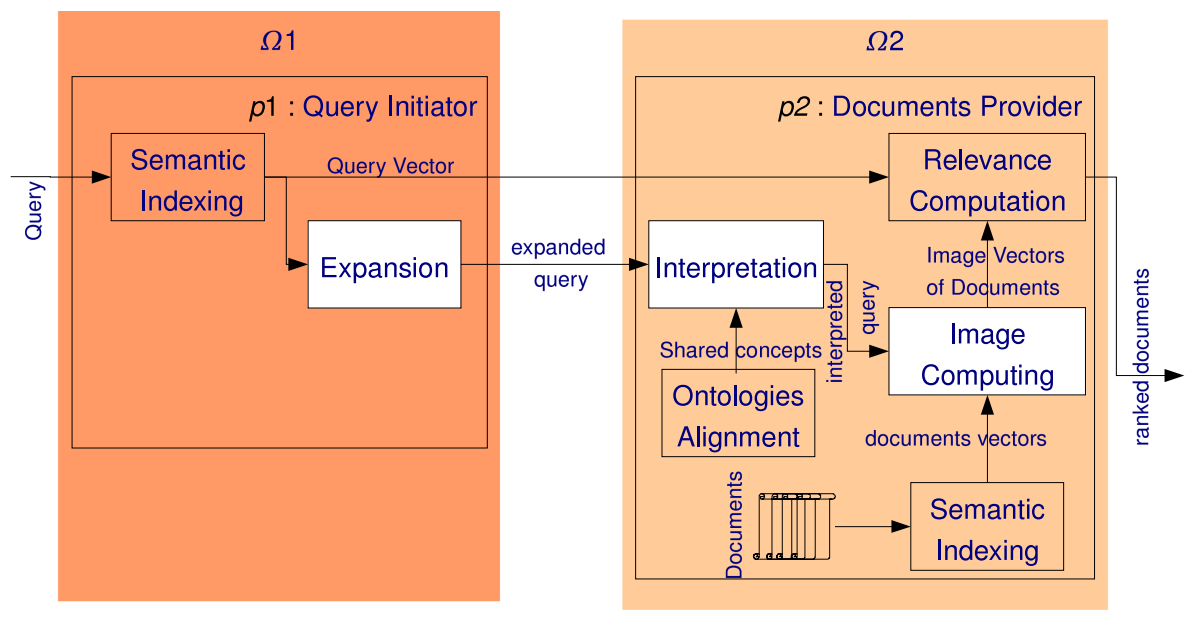

Fig. 4. Overview of relevance computation

Given these assumptions, computing relevance requires the following steps :

Query Expansion. It remains unchanged. The query initiator $p_{1}$ computes an expansion of its query, which results in a set of SEDs. Each SED is expressed on the set $\mathcal{C}_{\Omega_{1}}$, no matter the ontology used by $p_{2}$. Then, the expanded query is sent to $p_{2}$, together with the initial query.

Query Interpretation. Query interpretation by $p_{2}$ provides a set of interpreted SEDs on the set $\mathcal{C}_{\Omega_{2}}$ and an interpreted query. Each SED of the expanded query is interpreted separately. Interpretation of a SED $\overrightarrow{s e d}_{c}$ is decomposed in two problems, which we address in the next subsections:

- The first problem is to find a concept in $\mathcal{C}_{\Omega_{2}}$ that corresponds to c, noted $\tilde{c}$. This is difficult when the central concept is not shared. In this case, we use the weights of the shared concepts to guide the search. Of course, this is only a "contextual" correspondence as opposed to one that would be obtained through matching.

- The second problem is to attribute weights to shared and unshared concepts of $\mathcal{C}_{\Omega_{2}}$ which are linked to $\overrightarrow{s e d}_{c}$. This amounts to interpret the SED.

Image of the Document and Cosine Computation. They remain unchanged. Provider $p_{2}$ computes the image of its documents wrt. the interpreted SEDs and then, their cosine based relevance wrt. the interpreted query, no matter the ontology used by $p_{1}$.

In the following, we describe the steps involved in the interpretation of a given SED.

\subsection{Finding a Corresponding Concept}

The interpretation of a given SED $\overrightarrow{s e d}_{c}$ leads to a major problem: finding a concept in $\mathcal{C}_{\Omega_{2}}$ which corresponds to the central concept $c$. This corresponding 
concept is noted $\tilde{c}$ and will play the role of the central concept in the interpretation of $\overrightarrow{s e d}_{c}$, noted $\overrightarrow{s e d}_{\tilde{c}}$. If $c$ is shared, we just keep it as the central concept of the interpreted SED. When $c$ is not shared we have to find a concept which seems to best respect the "flavor" of the initial SED.

Theoretically, all the concepts of $\mathcal{C}_{\Omega_{2}}$ should be considered. Several criterias can apply to choose one which seems to best correspond. We propose to define the notion of interpretation function which is relative to a SED $\overrightarrow{s e d}_{c}$ and a candidate concept $\tilde{c}$ and which assigns a weight to each value of silmilarity wrt. $\tilde{c}$. Definition 4 consists of four points. The first one requires the interpretation function to assign the value of $\overrightarrow{\operatorname{sed}}_{c}[c]$ to the similarity value 1 , which corresponds to $\tilde{c}$. In the second point, we use the weights assigned by $\overrightarrow{s e d}_{c}$ to the shared concepts $\left(c_{1}, c_{2}, c_{3}\right.$ and $c_{6}$ in figure 5$)$ and the ranking of concepts in function of $\operatorname{sim}_{\tilde{c}}$. However, there might be several shared concepts that have the same similarity value wrt. $\tilde{c}$, but have a different weight according to $\overrightarrow{s e d}_{c}$. Thus, we require function $f_{i}^{\overrightarrow{s e d}_{c}, \tilde{c}}$ to assign the minimum of these values to the corresponding similarity value. This is a pessimistic choice and we could either take the maximum or a combination of these weights. As for the third point, let us call $c_{\text {min }}$, the shared concept with the lowest similarity value $\left(c_{6}\right.$ in Figure 5 (a) and $c_{3}$ in Figure 5 (b)). We consider that we have not enough information to weight the similarity values lower than $\operatorname{sim}_{\tilde{c}}\left(c_{\min }\right)$. Thus we assign them the zero value. The fourth point is just a mathematical expression which ensures that the segments of the affine function are only those defined by the previous points.

Definition 4 (Interpretation function). Given a SED $\overrightarrow{s e d}_{c}$ and a concept $\tilde{c}$, $f_{i}^{\overrightarrow{s e d}_{c}, \tilde{c}}:[0 . .1] \rightarrow[0 . .1]$, noted $f_{i}$ if no ambiguity, is an interpretation function iff it is a piecewise affine function and:

$$
\begin{aligned}
& -f_{i}(1)=\overrightarrow{\operatorname{sed}}_{c}[c] ; \\
& -\forall c^{\prime} \in \mathcal{C}_{\Omega_{1}} \cap \mathcal{C}_{\Omega_{2}}, f_{i}\left(\operatorname{sim}_{\tilde{c}}\left(c^{\prime}\right)\right)=\min \begin{array}{c}
\operatorname{sim}_{\tilde{c}}\left(c^{\prime}\right)=\mathcal{C}_{\Omega_{1}} \cap \mathcal{C}_{\Omega_{2}} \\
-\operatorname{sim}_{\tilde{c}}\left(c^{\prime \prime}\right)
\end{array} \\
& -\forall x \in[0 . .1], x<\operatorname{sim}_{\tilde{c}}\left(c_{\min }\right) \Rightarrow f_{i}[x)=0 ; \\
& - \text { Seg }=\|\left\{x: \exists c^{\prime \prime} \in \mathcal{C}_{\Omega_{1}} \cap \mathcal{C}_{\Omega_{2}}, c^{\prime} \neq \tilde{c} \text { and } \operatorname{sim}_{\tilde{c}}\left(c^{\prime}\right)=x\right\} \|+1 \text { where Seg is the } \\
& \quad \text { number of segments of } f_{i} .
\end{aligned}
$$

Intuitively, the criterias for choosing a corresponding concept among all the possible concepts can be expressed in terms of the properties of the piecewise affine function $f_{i}$. Of course, there are as many different function $f_{i}$ as candidate concepts. But the general idea is to choose the function $f_{i}$ wich resembles the more to a propagation function. Let us consider the example of Figure 5 (a) and (b) where $c_{1}, c_{2}, c_{3}$ and $c_{6}$ are shared. The function in Figure 5 (a) is obtained considering $c_{1}^{\prime}$ as the corresponding concept (and thus ranking the other concepts in function of their similarity with $c^{\prime} 1$ ). The function in Figure 5 (b) is obtained similarly, considering $c_{2}^{\prime}$. Having to choose between $c_{1}^{\prime}$ and $c_{2}^{\prime}$ we would prefer 
$c_{1}^{\prime}$ because function $f_{i}^{\overrightarrow{s e d}_{c}, c_{1}^{\prime}}$ is monotonically decreasing whereas $f_{i}^{\overrightarrow{s e d}_{c}, c_{2}^{\prime}}$ shows a higher "disorder" wrt. the general curve of a propagation function.

Several characteristics of the interpretation function can be considered to evaluate "disorder". For example, one could choose the function which minimizes the number of local minima (thus minimizing the number of times the sign of the derivated function changes). Another example is to choose the function which minimizes the variations of weight between local minima and their next local maximum (thus penalizing the functions which do not decrease monotonically). A third could combine these criteria.

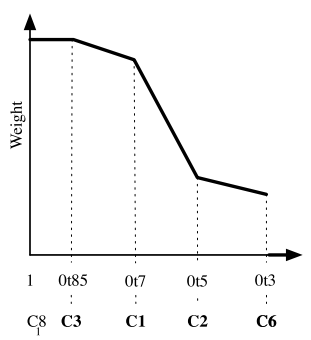

(a)

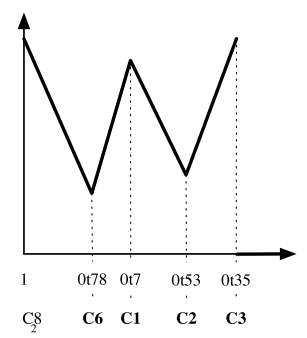

(b)

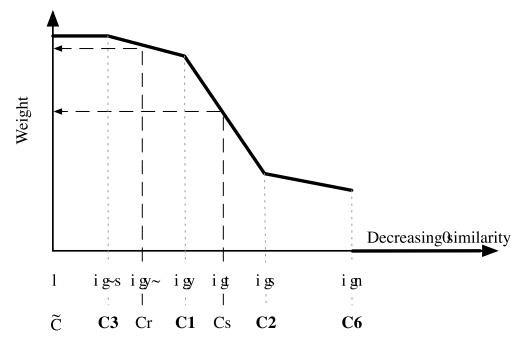

(c)

Fig. 5. Two steps of the interpretation of a SED : (a) $f_{i}$ for candidate concept $c_{1}^{\prime}$, (b) $f_{i}$ for candidate concept $c_{2}^{\prime}$ and (c) weighting the unshared concepts.

\subsection{Interpreting a SED}

We define the interpretation of a given SED $\overrightarrow{s e d}_{c}$ as another SED, with central concept $\tilde{c}$ which has been computed at the previous step. We keep their original weight to all the shared concepts. The unshared concepts are weighted using an interpretation function as defined above.

Definition 5 (Interpretation of a SED). Let $\overrightarrow{\operatorname{sed}}_{c}$ be a SED on $\mathcal{C}_{\Omega_{1}}$ and let $\tilde{c}$ be the concept corresponding to $c$ in $\mathcal{C}_{\Omega_{2}}$. Let $\operatorname{sim}_{\tilde{c}}$ be a similarity function

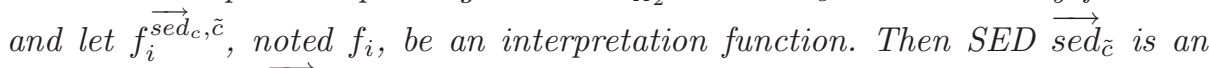
interpretation of $\overrightarrow{\operatorname{sed}}_{c}$ iff:

$$
\begin{aligned}
& -\overrightarrow{\operatorname{sed}}_{\tilde{c}}[\tilde{c}]=f_{i}(1) ; \\
& -\forall c^{\prime} \in \mathcal{C}_{\Omega_{1}} \cap \mathcal{C}_{\Omega_{2}}, \overrightarrow{\operatorname{sed}}_{\tilde{c}}\left[c^{\prime}\right]=\overrightarrow{\operatorname{sed}}_{c}\left[c^{\prime}\right] ; \\
& -\forall c^{\prime} \in \mathcal{C}_{\Omega_{2}} \backslash \mathcal{C}_{\Omega_{1}}, \overrightarrow{\operatorname{sed}}_{\tilde{c}}\left[c^{\prime}\right]=f_{i}\left(\operatorname{sim}_{\tilde{c}}\left(c^{\prime}\right)\right) ;
\end{aligned}
$$

Figure 5 (c) illustrates this definition. Document provider $p 2$ ranks its own concepts in function of $\operatorname{sim}_{\tilde{c}}$. Among these concepts, some are shared ones for which the initial SED $\overrightarrow{s e d}_{c}$ provides a given weight. This is the case for $c_{1}, c_{2}, c_{3}$ and $c_{6}$ which are in bold face in the figure. The unshared concepts are assigned the weight they obtain by function $f_{i}$ (through their similarity to $\tilde{c}$ ). This is illustrated for concepts $c_{4}$ and $c_{5}$ by a dotted arrow. 


\section{Experimental Validation}

In this section, we use our approach based on image based relevance to find documents which are the most relevant to given queries. We compare our results with those obtained by the cosine based method and the rough propagation method. In the former method, relevance is defined by the cosine between the query and document vectors. In the latter, the effects of propagating weights from different concepts are mixed in a single vector; then relevance is obtained using the cosine.

\subsection{General Setup for the Experiments}

We use the Cranfield corpus, a testing corpus consisting of 1400 documents and 225 queries in natural language, all related to aeronautical engineering. For each query, each document is scored by humans as relevant or not relevant (boolean relevance). Our ontology is lightweight, in the meaning of [7, i.e. an ontology composed of a taxonomy of concepts : WordNet [5]. In Information Retrieval, there was a debate whether WordNet is suitable for experimentation (see the discussion in 24]). However, more recent works show that it is possible to use WordNet, and sometimes other resources, and still get good results [8. Semantic indexing [20] is the process which can compute the semantic vectors from documents or queries in natural language. The aim is to find the most representative concepts for documents or queries. We use a program made in our lab : RIIO [3], which is based on the selection of synsets from WordNet. Although it is not the best indexing module, one of its advantages is that there is no human intervention in the process. The semantic similarity function we use is that of 2, because it has good properties and results which are discussed in Section [6] We slightly modified that function due to normalization considerations. Following the framework of membership functions presented in Section 2 we can define many propagation functions. We tested three different types of functions : "square" (of type $f_{v, l_{1}, l_{1}}$ ), "sloppy" (of type $f_{v, 1, l_{2}}$ ), or hybrid (of type $f_{v, l_{1}, l_{2}}$ with $l_{1}=2 \times l_{2}$ ). Our experiments show no important difference, but sloppy propagation has slightly better results. So we use only this propagation function, adding ten concepts in average for a given central concept.

In order to evaluate whether our solution is robust, we would need ontologies which agree on different percentages of concepts : $90 \%, 80 \%, 70 \%, \ldots, 10 \%$. This is very difficult to obtain. We could build artificial ontologies, but this would force us to give up the experiments on a real corpus. Thus, we decided to stick to WordNet and simulate semantic heterogeneity. Both the query initiator and the provider use WordNet, but we make so that they are not able to understand each other on some concepts (a given percentage of them). To do so, we remove some mappings between the two ontologies. Thus it simulates the case where the query intiator and the document provider use the same ontology but are not aware of it. It is then no more possible to compare queries and documents on those concepts. The aim is to evaluate how the answers to queries expressed with removed matchings, change. Note that the case with no removed matching reduces to a single ontology. 


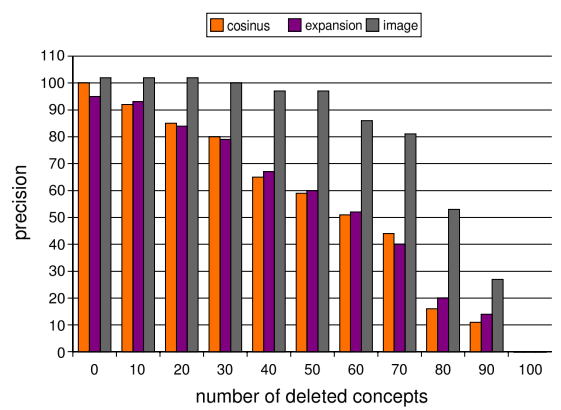

(a)

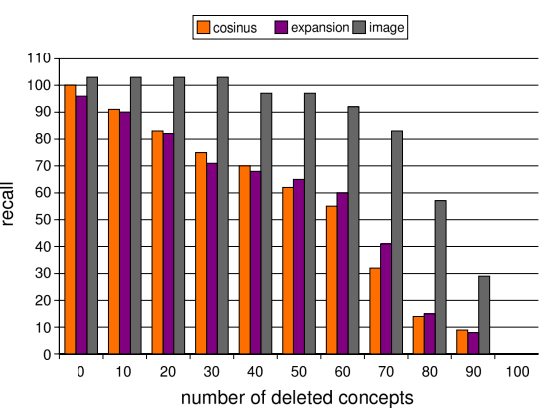

(b)

Fig. 6. Evolution of (a) precision and (b) recall in function of the random removal percentage of mappings

In a first experiment, we progressively reduce the number of mappings, thus increasing the percentage of removed mappings $(10 \%, 20 \%, \ldots$ until $90 \%)$. The progressive reduction in their common knowledge is done randomly. In a second experiment, we remove the mappings concerning the central concepts of the queries in the ontology of the document manager. This is now an intentional removing, which is the worst case for most of the techniques in IR : removing only the elements that match. For both experiments, we take into account the results obtained with the 225 queries of the corpus.

\subsection{Results}

Figure 6 shows the results obtained in average for the all 225 queries of the testing corpus. The reference method is the cosine one when no matching is removed, which gives a given reference precision and recall. Then, for each method and each percentage of removed matching, we compute the ratio of the precision obtained (respectively recall) by the reference precision. When the percentage of randomly removed matchings increases, precision (Figure 6 (a)) and recall (Figure 6 (b)) decrease i.e. the results are less and less relevant. However, our "image and interpretation based" solution shows much better results. When the percentage of removed matchings is under $70 \%$, we still get $80 \%$ or more of the answers obtained in the reference case.

In the second experiment, we consider that the document manager does not understand (i.e. share with the query initiator) the central concepts of the query (see Figure 7). With the cosine method, there is no more matching between concepts in queries and concepts in documents. Thus no relevant document could be retrieved. With the query expansion, some of the added concepts in the query allow to match with concepts in documents that are close to the central concepts of the query. This leads to precision and recall at almost 10\%. Our image-based retrieving method has more than $90 \%$ of precision and recall in the retrieval. This is also an important result. Obviously, as we have the same ontology and 


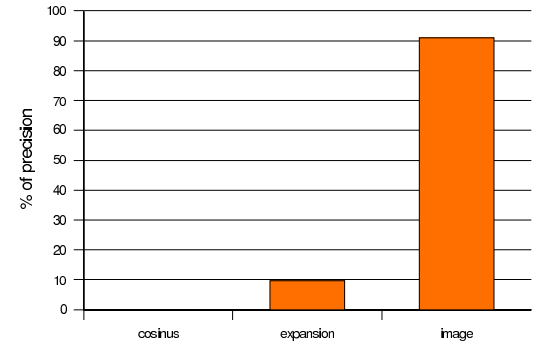

(a)

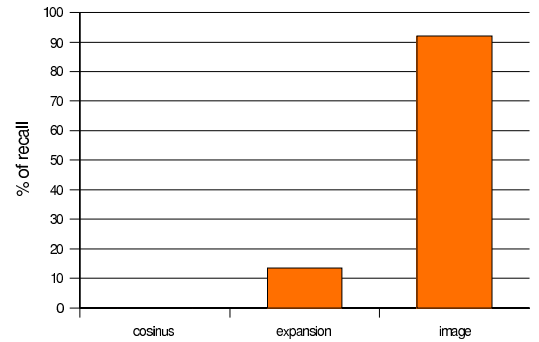

(b)

Fig. 7. Precision (a) and recall (b) when the central concepts of the query are unshared

the same similarity function, the interpretation can retrieve most of the central concepts of the query. But the case presented here is hard for most of the classical techniques (concepts of the query unshared) and we obtain a very important improvement.

\section{Related Work}

The similarity that we use in our experiments is the result of a thorough study of the properties of different similarity measures. We looked for a similarity which is not a distance (does not satisfy similarity nor triangle inequality), based on the result of 23 . Hence we use one classical benchmark of this domain : the work of Miller and Charles [15] on the human assessments of similarity between concepts. Thirty eight students were asked to mark how similar thirty couples of concepts were. We have implemented four similarity measures: [26 21,12], respectively noted $W u$ and $P$., Seco, Lin and Bidault in table 1, Correlation is the ratio between those measures on the human results. The results show that only Bidault's measure does not meet symmetry nor triangle inequality. Moreover, it obtains a slightly better correlation. Hence, it was preferred to rank the concepts according to their (dis)similarity with a central concept.

The idea of query expansion is shared by several fields. It was already used in the late 1980's in Cooperative Answering Systems [6]. Some of the suggested techniques expanded SQL queries considering a taxonomy. In this paper, we do not consider SQL queries, and we use more recent results about ontologies and

Table 1. Comparison of similarity measures

\begin{tabular}{|c|c|c|c|c|}
\hline & Wu \& P. & Seco & Lin & Bidault \\
\hline symmetry & yes & yes & yes & no \\
\hline triangle inequality & no & no & no & no \\
\hline correlation & 0.74 & 0.77 & 0.80 & 0.82 \\
\hline
\end{tabular}


their interoperability. Expansion of query vectors is used for instance in [17/24]. However, this expansion produces a single semantic vector only. This amounts to mix the effects of the propagations from different concepts of the query. Although this method avoids some silence, it often generates too much noise, without any highly accurate sense disambiguation 24. Consequently, the results can be worse than in the classical vector space model [1]. Our major difference with this approach is that (1) the propagations from the concepts of the query are kept separate and that (2) they are not directly compared with the document. Rather, they are used to modify its semantic vector. In our experiments, our method gives better results. Also, we join [16] on their criticism of the propagation in a single vector, but our solutions are different.

Our approach also relies on the correspondences resulting from the matching of the two ontologies. Several existing matching algorithms could be used in our case [4. In the interpretation step, we provide a very general algorithm to find the concept corresponding to the central concept of a SED. In case the concept is not shared, one could wonder whether matching algorithms could be used. In the solution we propose, the problem is quite different because the weights of the concepts are also used to find the corresponding concept (through the interpretation function). This is not the case in traditional ontology matching, which aim is to find general correspondences. In our case, one can see the problem as finding a "contextual" matching, the results of which cannot be used in other contexts. Because it is difficult to compute all the interpretation functions, one can use an approximation algorithm (for example, taking the least common ancestor as we did in our experiments). In that case, existing proposals can fit like 1014 . But it is clear that they do not find the best solution every time.

Finally, the word interpretation is used very often and reflects very different problems. However, to the best of our knowledge, it never refers to the case of interpreting a query expressed on some ontology, within the space of another ontology, by considering the weights of the concepts.

\section{Conclusion}

The main contribution of this paper is a proposal improving information exchange between a query initiator and a document provider that use different ontologies, in a context where semantic vectors are used to represent documents and queries. The approach only requires the initiator and the provider to share some concepts and also uses the unshared ones to find additional relevant documents. To our knowledge, the problem has never been addressed before and our approach is a first, encouraging solution. In short, when performing query expansion, the query initiator makes more precise the concepts of the query by associating an expansion to each of them (SED). The expansion depends on the initiator's characteristics: ontology, similarity, propagation function. However, as far as shared concepts appear in a SED, expansion helps the document provider interpreting what the initiator wants, especially when the central concept is not shared. Interpretation by the document provider is not easy because the peers 
do not share the same vector space. Given its own ontology and similarity function, it first finds out a correspondent concept for the central concept of each SED, and then interprets the whole SED. The interpreted SEDs are used to compute an image of the documents and their relevance. This is only possible because the central concepts are expanded separately. Indeed if the effects of propagations from different central concepts were mixed in a single vector, the document provider wouldn't be able to interpret the query as precisely.

Although our approach builds on several notions (ontology, ontology matching, concept similarity, semantic indexing, relevance of a document wrt a query... ) it is not stuck to a specific definition or implementation of them and seems compatible with many instantiations of them. It is important to notice that there is no human intervention at all in our experiments, in particular for semantic indexing. Clearly, in absolute, precision and recall could benefit from human interventions at different steps like indexation or the definition of the SEDs. Results show that our approach significantly improves the information exchange, finding up to $90 \%$ of the documents that would be found if all the concepts were shared.

As future work, we plan to test our approach in several different contexts in order to verify its robustness. Many different parameters can be changed: similarity and propagation functions, ontologies, indexing methods, corpus... Complexity is another point that should be considered carefully. Indeed, naive implementations would lead to unacceptable execution time. Although an implementation is running for the experiments within admissible times, it could benefit from a more thorough study of theoretical complexity.

\section{References}

1. Berry, M.W., Drmac, Z., Jessup, E.R.: Matrices, vector spaces, and information retrieval. SIAM Rev. 41(2) (1999)

2. Bidault, A., Froidevaux, C., Safar, B.: Repairing queries in a mediator approach. In: ECAI (2000)

3. Desmontils, E., Jacquin, C.: Indexing a web site with a terminology oriented ontology. In: The Emerging Semantic Web (2002)

4. Euzenat, J., Shvaiko, P.: Ontology matching. Springer, Heidelberg (DE) (2007)

5. Fellbaum, C.: WordNet: an electronic lexical database (1998)

6. Gaasterland, T., Godfrey, P., Minker, J.: An overview of cooperative answering. J. of Intelligent Information Systems 1(2), 123-157 (1992)

7. Gómez-Pérez, A., Fernández, M., Corcho, O.: Ontological Engineering. Springer, London (2004)

8. Gonzalo, J., Verdejo, F., Chugur, I., Cigarran, J.: Indexing with wordnet synsets can improve text retrieval. In: COLING/ACL 1998 Workshop on Usage of WordNet for NLP (1998)

9. Ives, Z.G., Halevy, A.Y., Mork, P., Tatarinov, I.: Piazza: mediation and integration infrastructure for semantic web data. Journal of Web Semantics (2003)

10. Jiang, G., Cybenko, G., Kashyap, V., Hendler, J.A.: Semantic interoperability and information fluidity. Int. J. of cooperative Information Systems 15(1), 1-21 (2006) 
11. Jiang, J., Conrath, D.: Semantic similarity based on corpus statistics. In: International Conference on Research in Computational Linguistics (1997)

12. Lin, D.: An information-theoretic definition of similarity. In: International Conf. on Machine Learning (1998)

13. Manning, C.D., Schtze, H.: Foundations of statistical natural language processing. MIT Press, Cambridge (1999)

14. Mena, E., Illaramendi, A., Kashyap, V., Sheth, A.: Observer: An approach for query processing in global information sytems based on interoperation across preexisting ontologies. Int. J. distributed and Parallel Databases 8(2), 223-271 (2000)

15. Miller, G.A., Charles, W.G.: Contextual correlates of semantic similarity. Language and Cognitive Processes (1991)

16. Nie, J.-Y., Jin, F.: Integrating logical operators in query expansion invector space model. In: SIGIR workshop on Mathematical and Formal methods in Information Retrieval (2002)

17. Qiu, Y., Frei, H.P.: Concept based query expansion. In: SIGIR (1993)

18. Rousset, M.-C.: Small can be beautiful in the semantic web. In: International Semantic Web Conference, pp. 6-16 (2004)

19. Salton, G., MacGill, M.J.: Introduction to Modern Information Retrieval. McGrawHill, New York (1983)

20. Sanderson, M.: Retrieving with good sense. Information Retrieval (2000)

21. Seco, N., Veale, T., Hayes, J.: An intrinsic information content metric for semantic similarity in wordnet. In: ECAI (2004)

22. Tempich, C., Pinto, H.S., Staab, S.: Ontology engineering revisited: An iterative case study. In: ESWC, pp. 110-124 (2006)

23. Tversky, A.: Features of similarity. Psychological Review 84(4) (1977)

24. Voorhees, E.M.: Query expansion using lexical-semantic relations. In: SIGIR, Dublin (1994)

25. Woods, W.: Conceptual indexing: A better way to organize knowledge. Technical report, Sun Microsystems Laboratories (1997)

26. Wu, Z., Palmer, M.: Verb semantics and lexical selection. In: ACL (1994) 